\title{
Subthalamic electrode implantation using the MicroDrive system and the importance of microrecording data
}

\author{
Chrastina $\mathrm{J}^{1}$, Novak $\mathrm{Z}^{2}$, Balaz $\mathrm{M}^{2}$, Riha $\mathrm{I}^{1}$, Bockova $\mathrm{M}^{2}$ \\ Department of Neurosurgery MF MU FH St Ann's, Brno, Czech Republic. jan.chrastina@fnusa.cz
}

\begin{abstract}
Objective: The aim of the paper was to describe the relationship of the anatomical and electrophysiological target for the subthalamic electrode implantation in Parkinson's disease patients defined as the best electrophysiological recordings from multiple paralel electrodes tracts with a target discrepancy explanation. Background: Although microrecording is the standard in subthalamic stimulation, microelectrode monitoring prolongs surgical time and may increase the risk of haemorrhagic complications. The main purpose for the electrophysiological mapping is to overcome the discrepancy between the anatomical and electrophysiological targets. Methods: Subthalamic electrodes were stereotactically implanted in 58 patients using microrecording by means of parallel electrodes at defined distances. The relationship of the final electrode to the anatomical trajectory, the subthalamic nucleus electrical activity length, and the relationship of right and left electrodes were analysed. Results: The final electrode placement matched the anatomical trajectory in $53.4 \%$ of patients on the right side, and $43.1 \%$ of patients on the left side. The electrode position was symmetrical in $38.3 \%$ of patients. The analysis of left and right electrode positions did not prove brain shift as the sole factor responsible for anatomyfunctional discrepancy. Further, neither age, Parkinson's disease duration, or L-DOPA adverse effects were confirmed as responsible factors.

Conclusions: The difference between the anatomical trajectory and the final electrode placement underlined the need for functional microelectrode monitoring. Brain shift is not the only causative factor for the difference (Tab. 7, Ref. 27). Full Text in PDF www.elis.sk.

Key words: stereotaxy, subthalamic stimulation, microelectrode recording, brain shift.
\end{abstract}

Both direct and indirect targeting techniques can be used for deep brain stimulation electrode implantation into the subthalamic nucleus in Parkinson's disease patients. The principle of indirect subthalamic nucleus targeting utilises the known spatial relationship of the surgical target and the intercommissural line or structures that can be easily visualised (e.g. red nucleus for subthalamic electrode targeting). The direct targeting technique relies on direct visualisation of the target structure $(1,2)$.

The results of anatomical targeting can be supported by electrophysiological techniques that include monitoring the target and its surroundings and stimulation in order to verify the target structure. The use of micromonitoring techniques is well established and typical recordings of subthalamic electrophysiological activity as recorded by microelectrodes support the use of electrophysiological recordings in subthalamic stimulation. According to a survey of North American centres implanting deep brain stimulation (DBS)

'Department of Neurosurgery MF MU FH St Ann's, Brno, Czech Republic, and 'Ist Department of Neurology MF MU FH St Ann's, Brno, Czech Republic

Address for correspondence: J. Chrastina, MD, PhD, Department of Neurosurgery MF MU FH St Ann's Brno, Pekarska 53, CZ-656 91 Brno, Czech Republic.

Phone: +420.5.43182697, Fax: +420.5 .43182687$

Acknowledgement: Supported by VZ MSMT 0021622404 and MZCR IGA NS10411-3. electrodes, microelectrode monitoring was used in $97 \%$ of the participating centres (3). However, the overall review of literature conducted by Hariz revealed that microelectrode monitoring does not necessarily improve the clinical outcome or targeting results in comparison with macrostimulation or impedance monitoring (4). Microelectrode monitoring may also prolong the length of surgery. The risk of haemorrhagic complications caused by multiple penetration of microelectrodes (simultaneous or sequential) cannot be ignored $(5,6,7)$. The risk of haemorrhage is reported to be higher for pallidal (6.7\%) than subthalamic (2.7\%) surgeries (8). Papers presenting excellent surgical outcomes without electrophysiological monitoring should also be considered (9), including data published about deep brain electrode implantation in interventional MRI without electrophysiological monitoring (10).

More or less sophisticated techniques are used to define the relationship between the final target structures selected by electrophysiological techniques and the anatomically-defined target. The distance between the anatomical target and the final target can be defined as the difference between the target structure and the optimum contact position in $\mathrm{x}, \mathrm{y}, \mathrm{z}$ coordinates or as a scalar or vector distance between the optimum contact position and the planned target $(2,11)$. A more simple technique of anatomical and functional target analysis is based on the fact that systems enabling monitoring electrode implantation (e.g. MicroDrive) in parallel directions in defined interelectrode distances are used for 
microelectrode monitoring and final electrode implantation. The result of the intraoperative electrophysiological monitoring performed with this system is defined as the optimum trajectory as related to system ports for electrode implantation and the length of subthalamic nucleus electrophysiological recordings. Although the technique may look oversimplified for statistical studies, the relationship between the anatomical target and the electrophysiological target can be studied and the cause of possible differences can be defined.

\section{Methods}

Between 2003 and 2009, 59 patients (39 males, 19 females) suffering from motor complications of advanced Parkinson's disease underwent implantation of 117 subthalamic DBS electrodes (Tab. 1). With the exception of one patient (a 59-year-old male with transient cognitive disturbance and predominantly unilateral problems), all patients underwent bilateral implantation.

A ceramic MRI-compatible Leibinger stereotactic frame was attached to the patient's head under a local anaesthesia on the morning of surgery. A combination of direct and indirect techniques was used to define the subthalamic nucleus coordinates. The imaging protocol include T2-weighted fat-saturation study in the axial and coronal planes, MRI angiography (arterial and venous phase), and 3D gradient echo multiplanar reconstruction T1-weighted image (WI) after a contrast administration. The image sets were merged in a dedicated computer planning workstation using stereotactic planning software (Praezis Plus, Tatramed, Slovakia). The initial coordinates for the subthalamic nucleus (dorsolateral) were then determined in reference to the anterior commissure-posterior commissure (AC-PC) line (intercommissural line) using an indirect technique, typically $11 \mathrm{~mm}$ lateral, $3 \mathrm{~mm}$ posterior, and $5 \mathrm{~mm}$ ventral to the intercommissural point. The final target coordinates were then modified with respect to direct visualisation of the subthalamic nucleus on T2-WI and in relations to the red nucleus anterior margin and the largest red nucleus cross-sectional area. The distance from the red nucleus centre can be also considered (approximately 3.5 to $4 \mathrm{~mm}$ in front of the centre of the red nucleus and $6.5 \mathrm{~mm}$ laterally). A particular attention was paid to the posterior cerebral artery and its branches during target and trajectory planning, because the vessels may form loops, especially in patients of advanced age, and prevent safe electrode implantation. Similar attention was paid to intraventricular veins and cortical vessels.

The intraoperative microelectrode recording together with the intraoperative stimulation using the MicroDrive system were used to confirm the results of anatomical targeting. The principle

\section{Tab. 1. Parkinson's disease patients - group description.}

\begin{tabular}{lc}
\hline Parameter & Descriptive statistics \\
\hline Number of patients & 58 \\
Age & $62.0(38.0 ; 74.0)$ \\
Females & $19(32.8 \%)$ \\
Parkinson's disease duration ( years) & $11.0(5.0 ; 24.0)$ \\
Side effects duration (years) & $4.0(1.0 ; 13.0)$ \\
\hline continuous variables represented by median, minimum and maximum values; binary \\
/categorical variables are represented by percentages
\end{tabular}

of the MicroDrive system is the simultaneous implantation of up to 5 parallel microelectrodes in defined positions related to a central trajectory (anatomical trajectory), marked anterior $(2.5 \mathrm{~mm}$ anterior to the central trajectory), lateral $(2.5 \mathrm{~mm}$ lateral to the central trajectory), medial (2.5 mm medial to the central trajectory) and posterior ( $2.5 \mathrm{~mm}$ posterior to the central trajectory). For subthalamic electrode implantation, a combination of central, anterior, lateral, and posterior electrodes was used in all patients for the intraoperative microelectrode recording. Microelectrode recording was started $10 \mathrm{~mm}$ above the anatomical target and microelectrodes were advanced in $1 \mathrm{~mm}$ steps until $5 \mathrm{~mm}$ above the anatomical target $(0.5 \mathrm{~mm}$ steps $)$. The subthalamic activity was recorded for each electrode. The length of the subthalamic nucleus was defined as the distance between the points where the subthalamic activity was first recorded to the end of recorded subthalamic activity for each side.

The motor part of the subthalamic nucleus was identified by recording the pattern of neuronal activity, background activity, and motor responsiveness (changes in neuronal firing in response to passive and active manipulation of contralateral limbs during the perioperative microrecording). We determined the dorsolateral border of the subthalamic nucleus by the microelectrode recording of cellular firing patterns. We also assessed the lead location by evaluating the symptom response to the intraoperative microstimulation.

After the completion of microelectrode monitoring, the intraoperative stimulation by means of the electrodes with the best microelectrode recording was performed, and the effect of stimulation on rigidity, tremor, and bradykinesis was monitored. Because these changes might not be apparent when observed intraoperatively, another purpose of the intraoperative stimulation is to identify possible adverse events, such as speech changes, paresthesia (transient paresthesias do not contraindicate electrode implantation because their cause may be the stimulation of the posterior subthalamus) and eye deviation (conjugate deviation caused by corticospinal tract stimulation, and dysconjugate deviation caused by oculomotor nucleus stimulation). Elicitation of dyskinesia by the intraoperative stimulation of the subthalamic nucleus area can also be assessed.

The Zamorano-Dujovny stereotactic system was used for electrode implantation in all patients, and the right-sided electrode was the first electrode implanted in all patients.

Fluoroscopic images were taken at the moment of microelectrode guiding tube implantation, before the start of monitoring and during a definitive electrode implantation to exclude any deviation of the electrode guiding tubes and electrodes. The final lead position was verified by postoperative stereotactic CT, which also aimed to exclude postoperative complications.

\section{Results}

There was no symptomatic intracerebral haematoma requiring surgical intervention. One asymptomatic small subcortical haematoma $(1.5 \mathrm{~cm})$ immediately beneath the trephination opening was found on a postoperative CT in one asymptomatic patient. 
Tab. 2. Location of implanted electrode as related to MicroDrive trajectories on the left and right sides.

\begin{tabular}{lccc}
\hline Electrode $^{1}$ & Left side $(\mathrm{n}=58)$ & Right side $(\mathrm{n}=58)$ & $\mathrm{p}^{2}$ \\
\hline Central & $43.1 \%$ & $53.4 \%$ & 0.353 \\
Posterior & $24.1 \%$ & $25.9 \%$ & 0.999 \\
Anterior & $17.2 \%$ & $13.8 \%$ & 0.798 \\
Lateral & $12.1 \%$ & $1.7 \%$ & 0.061 \\
\hline
\end{tabular}

Tab. 3. Relationship of left and right electrodes.

\begin{tabular}{lc}
\hline Left to right electrode & Number of patients \\
\hline Posterior shift $2.5 \mathrm{~mm}$ & 11 patients \\
Posterior shift $5 \mathrm{~mm}$ & 2 patients \\
Lateral shift $2.5 \mathrm{~mm}$ & 4 patients \\
Anterior shift $2.5 \mathrm{~mm}$ & 12 patients \\
Medial shift $2.5 \mathrm{~mm}$ & 1 patient \\
Anterior and lateral shift $2.5 \mathrm{~mm}$ & 3 patients \\
Posterior and lateral shift $2.5 \mathrm{~mm}$ & 2 patients \\
Anterior shift $5 \mathrm{~mm}$ & 2 patients \\
Medial and anterior shift 2.5 & 1 patient \\
\hline
\end{tabular}

Tab. 4. Microrecording-defined subthalamic nucleus activity length (median).

\begin{tabular}{ll}
\hline Subthalamic nucleus length - right side $(\mathrm{mm})$ & $3.5(2.0 ; 6.0)$ \\
Subthalamic nucleus length - left side $(\mathrm{mm})$ & $4.0(2.0 ; 7.0)$ \\
\hline
\end{tabular}

The position of the implanted electrode as defined by the MicroDrive parallel trajectories is presented in the Table 2. In all patients, the final electrode matched the trajectory with the longest recorded subthalamic activity. On the right side, the final electrode was implanted in the central position in $53.4 \%$ of patients. On the left side, the percentage of central electrodes was $43.1 \%$. However, the difference of electrode percentages implanted in individual positions does not reach the level of statistical significance (Fischer exact test), not only for central (anatomical trajectory) but also for other electrode trajectories (posterior, anterior, and lateral).The position of implanted electrodes was symmetrical in $38.3 \%$ of patients and asymmetrical in $61.7 \%$ of patients. Although the intraoperative stimulation was performed in all our patients, the results of the intraoperative stimulation did not alter the final electrode trajectory.

Table 3 compares the positions of definite electrodes implanted on the right (first implanted) and left sides (implanted after a right-sided surgery) in the plane perpendicular to the long axis of the MicroDrive system.

Table 4 presents the length of recorded subthalamic nucleus activity for right and left sides.

The length of the recorded subthalamic activity in the central electrode was 2 to $5 \mathrm{~mm}$ (median $3 \mathrm{~mm}$ ) on the right side and 2 to $6 \mathrm{~mm}$ (median $3.5 \mathrm{~mm}$ ) on the left side. In patients with implanted other than central electrode, the length of subthalamic activity was 2 to $6 \mathrm{~mm}$ (median $4 \mathrm{~mm}$ ) on the right side and 2 to $7 \mathrm{~mm}$ (median $4 \mathrm{~mm}$ ) on the left side. For left subthalamic electrodes, a statistical evaluation did not prove a difference of subthalamic activity length between the central electrodes and other electrode locations. In right subthalamic electrodes, the statistical evaluation proved a significantly longer subthalamic activity in other than the central electrode locations (Tab. 5).

The patient age, duration of Parkinson's disease, and L-DOPA treatment adverse effects were compared between the group of patients with implanted central electrodes and the group with other definitive electrode locations. A statistical evaluation did not confirm a significant difference between the ages of patients with central electrodes and patients with non-central electrodes implanted for both right and left electrode. Similarly, a statistical evaluation did not confirm a longer duration of Parkinson's disease or L-DOPA treatment adverse effects in the patients with non-central electrodes implanted for both sides (Tab. 6).

Tab. 5. Lengths of subtalamic recordings.

\begin{tabular}{|c|c|c|c|c|c|c|}
\hline \multirow[b]{2}{*}{$\underline{\text { Parameter1 }}$} & \multicolumn{3}{|c|}{ Left } & \multicolumn{3}{|c|}{ Right } \\
\hline & Central & Other & $\mathrm{p}^{2}$ & Central & Other & $\mathrm{p}^{2}$ \\
\hline Length of subthalamic nucleus (mm) & $3.5(2.0 ; 6.0)$ & $4.0(2.0 ; 7.0)$ & 0.337 & $3.0(2.0 ; 5.0)$ & $4.0(2.0 ; 6.0)$ & 0.036 \\
\hline
\end{tabular}

Tab. 6. Patients with final central electrode compared with other electrode location.

\begin{tabular}{|c|c|c|c|c|c|c|}
\hline \multirow[b]{2}{*}{$\underline{\text { Parameter }}$} & \multicolumn{3}{|c|}{ Left side } & \multicolumn{3}{|c|}{ Right side } \\
\hline & Central & Other & $\mathrm{p}^{2}$ & Central & Other & $\mathrm{p}^{2}$ \\
\hline Number of patients & 25 & 33 & & 31 & 27 & \\
\hline Females & $40.0 \%$ & $27.3 \%$ & 0.399 & $41.9 \%$ & $22.2 \%$ & 0.162 \\
\hline Age(years) & $60(47 ; 68)$ & $62(38 ; 74)$ & 0.887 & $63(50 ; 69)$ & $60(38 ; 74)$ & 0.214 \\
\hline Disease duration (years) & $10(5 ; 24)$ & $11(5 ; 22)$ & 0.518 & $11(7 ; 19)$ & $10(5 ; 24)$ & 0.452 \\
\hline L DOPA treatment adverse effect duration (years) & $4(1 ; 12)$ & $4(1 ; 13)$ & 0.651 & $4(1 ; 13)$ & $4(1 ; 11)$ & 0.474 \\
\hline
\end{tabular}

Tab. 7. Patients with final central electrode compared with posterior electrode (second most frequently implanted).

\begin{tabular}{|c|c|c|c|c|c|c|}
\hline \multirow[b]{2}{*}{ Parameter $^{1}$} & \multicolumn{3}{|c|}{ Left side } & \multicolumn{3}{|c|}{ Right side } \\
\hline & Central & Posterior & $\mathrm{p}^{2}$ & Central & Posterior & $\mathrm{p}^{2}$ \\
\hline Number of patients & 25 & 14 & & 31 & 15 & \\
\hline Females & $40.0 \%$ & $35.7 \%$ & 0.999 & $41.9 \%$ & $20.0 \%$ & 0.195 \\
\hline Age & $60(47 ; 68)$ & $59(38 ; 69)$ & 0.409 & $63(50 ; 69)$ & $56(38 ; 74)$ & 0.119 \\
\hline Disease duration (years) & $10(5 ; 24)$ & $12(5 ; 19)$ & 0.496 & $11(7 ; 19)$ & $10(5 ; 22)$ & 0.480 \\
\hline L DOPA treatment adverse effect duration (years) & $4(1 ; 12)$ & $4.5(1 ; 13)$ & 0.965 & $4(1 ; 13)$ & $4(1 ; 11)$ & 0.329 \\
\hline Subthalamic nucleus length (mm) & $3.5(2.0 ; 6.0)$ & $4.5(2.0 ; 7.0)$ & 0.054 & $3,0(2,0 ; 5,0)$ & $4.0(2.0 ; 6.0)$ & 0.043 \\
\hline
\end{tabular}


311-316

The posterior trajectory was the second most frequently implanted after the central-anatomical trajectory. The results of statistical analysis comparing the patient age and Parkinson's disease duration between patients with central or posterior electrodes did not confirm a significant difference between the groups. However, a statistical analysis confirmed a significantly longer subthalamic nucleus recording in patients with the posterior right-sided electrodes than in patients with the central right-sided electrodes (Tab. 7).

\section{Discussion}

The need for the intraoperative electrophysiological monitoring is strongly supported by the difference between the anatomical target structure and the final target structure as defined by the electrophysiological intraoperative microrecording and macrostimulation, not only in subthalamic stimulation.

For instance, the paper published by Foerster et al. showed that the electrophysiological monitoring (microelectrode monitoring, evoked potentials, and macrostimulation) modified the surgery in 67 $\%$ of cases (mainly thalamic targets), but the authors declared that more sophisticated planning techniques resulted in a better correlation between the anatomical and electrophysiological targets (12).

Recently, in a group of 42 patients operated on for dystonia (pallidal stimulation), Pinsker implanted the central-anatomical electrode in $64 \%$ of the patients, even when the target structure was defined using stereotactic T2 or inversion recovery MRI. The medial trajectory was optimal in $20 \%$ of the patients; the anterior in $9 \%$, and the lateral with posterior trajectories in $3.5 \%$ each [13].

A similar technique comparing the anatomical target and final electrode target as defined by microelectrode recording was used in the Amirnovin's study of a group of 70 subthalamic electrodes implanted in 40 patients with a target confirmation using intraoperative monitoring. Although the system used for the electrode implantation and intraoperative monitoring was not MicroDrive (3 parallel ports for microelectrodes and final electrodes $2 \mathrm{~mm}$ apart), the results are comparable. Their results showed a slightly increased incidence of intracerebral haematomas (4 out of 40 patients). The frequency of electrode trajectories used for final electrode implantation was anterior $29 \%$, central-anatomical $42 \%$, and $29 \%$ posterior on the right side; and anterior $35 \%$, central-anatomical $39 \%$, and $26 \%$ posterior on the left side. Interestingly, the authors began with the left electrode in bilateral surgeries. The percentage of the central-anatomical electrodes did not differ between the left and right sides, although there was a tendency towards a lower percentage of anatomical electrodes on the first implanted left side (14). On the other hand, in the paper by Hamid et al, the authors found it easier to define the subthalamic nucleus electrical activity on the side first operated on, although their monitoring technique was different (not using simultaneous implantations of several parallel electrodes; when the recording obtained was not satisfactory, the electrode was removed and reinserted $2 \mathrm{~mm}$ from the initial target) (15).

Similarly, Bour implanted the anatomical-central electrode in $50 \%$ of patients with subthalamic nucleus stimulation. In 64 $\%$ of the cases, the channel selected for the permanent electrode implantation corresponded with the trajectory with the longest recorded subthalamic activity. The subthalamic microrecording was considered to be the indicator of the best electrode for the intraoperative macrostimulation and the final electrode implantation. Similar data were obtained for pallidal surgeries (16).

Brain shift may be one possible explanation for the difference between the anatomical target structure and the final target for electrode implantation as adjusted according to the results of the electrophysiological monitoring. However, other causes of the difference between the anatomical target and the final electrode placement may include the degree of target determination accuracy (the difference between the target locations using different targeting methods) (11), MRI image distortion (15), technical aspects of electrode implantation, and interindividual variants of subthalamic nucleus shape and size. The anatomical position of the subthalamic nucleus may not strictly follow the AC-PC line due to its complex embryological origin. Its medial part derives from the lateral hypothalamus, whereas the lateral part is linked to the development of the pallidum (17). More recently, important genetic factors playing role in the development of Parkinson's disease were recognised (18). However, the association between the identified genetic factors and intraoperative findings was not described so far. In a study evaluating the variability of the subthalamic nucleus and the coordinates of its centre, Daniluk et al. showed that the subthalamic nucleus lateral coordinate ranged from 8.7 to $14.5 \mathrm{~mm}$, the anteroposterior coordinate from $3.5 \mathrm{~mm}$ posterior to $0.5 \mathrm{~mm}$ anterior, and the vertical coordinate from 1.3 to $6 \mathrm{~mm}$ below the midcommissural point. The lateral coordinate strongly correlated with the width of the third ventricle (19).

Nunta-aree et al. compared four different targeting techniques for the subthalamic nucleus, with the average of the final lead position of each technique used to determine an appropriate AC-PC based formula for the subthalamic nucleus in Thai patients. The appropriate formula was $11.5 \mathrm{~mm}$ lateral, $3.5 \mathrm{~mm}$ posterior, and $5.0 \mathrm{~mm}$ inferior to the mid-commissural point, which only slightly differed from the initial target coordinates used for the subthalamic nucleus targeting in our patients (20).

Brain shift may occur any time between imaging study acquisition and fthe inal electrode implantation. The cause of brain shift or brain sink may not be only the loss of cerebrospinal fluid with resulting pneumocephalus, but also the gravitational movements of brain tissue depending on head position (for instance, planning data acquisition taking place in a different position than the surgical position, i.e., patient lying flat versus slight head elevation). Brain tissue movements may also be caused by brain deformation during temporary or final electrode implantation (21).

Because indirect techniques of subthalamic nucleus targeting employ the relationship between the target structure and the intercommissural line, the problem of the commissural shift during stereotactic surgery has been discussed by many authors.

Miyagi et al proved a higher degree of AC-PC shifts in patients with bilateral implants than in patients with unilateral implants: $2.2 \mathrm{~mm}$ (SD - Standard Deviation $-0.9 \mathrm{~mm}$ ) median AC shift in bilateral implants and $1.2 \mathrm{~mm}$ (SD $0.9 \mathrm{~mm}$ ) median shift of AC in unilateral implants. The median value of the PC shift was lower: 0.7 
$\mathrm{mm}(\mathrm{SD} 0.6 \mathrm{~mm})$ in unilateral implants and $1.0 \mathrm{~mm}(\mathrm{SD} 0.9 \mathrm{~mm})$ in bilateral implants. The difference was explained by air entering the subdural space with a resulting brain shift. Air entry after the first electrode implantation should result in a contralateral brain shift. After the contralateral trephination for the second electrode implantation, shift equilibrium is established because of air entry from the contralateral side (22). However, the support by the contralateral hemisphere is lost and a significant caudal brain shift occurs. When applying their conclusions to data describing the final electrode location, the lateral and posterior final electrode targets resulting from posterior or lateral electrode shifts should prevail in left-sided, rather than right-sided, electrodes. This assumption was not confirmed, although there was some tendency for a larger percentage of lateral electrodes on the left side.

In the study by Elias, the extent of commissural shift was higher than in the Miyagi study, varying between -5.6 to $1 \mathrm{~mm}$ for AC and -1.7 to $0.5 \mathrm{~mm}$ for PC. The authors found a significant correlation between the extent of postoperative pneumocephalus and AC shift (23). In Petersen's paper, the AC-PC shift was less marked (AC $-0.4 \pm 0.6 \mathrm{~mm}$ and $\mathrm{PC}-0.2 \pm 0.7 \mathrm{~mm}$ ) (21).

Winkler et al studied the association between the degree of frontal cortex and subthalamic nucleus shift using a tissue deformation technique. A frontal cortex shift of $-12 \mathrm{~mm}$ was associated with a $2 \mathrm{~mm}$ subthalamic nucleus shift. Morphological results were supported by microelectrode data (24).

Because the right electrode was the first to be implanted in all patients, the percentage of central trajectories was expected to be lower on the left side as the result of brain shift. Although the percentage of central trajectories was higher on the right side, the statistical evaluation did not prove a significant difference. In the study by Amirnovin, the left-sided electrode was implanted first, but the percentage of central electrodes was surprisingly slightly higher on the right side, at $42 \%$, than on the left side, at $39 \%$ (14). Bour et al noted a non-significant trend towards a less frequent choice for the central electrode on the side operated on second (right side). The authors attribute the difference to cerebrospinal fluid loss and subdural air invasion with potential brain displacement. Although the authors used a medial electrode port for monitoring, medial electrodes were implanted in only $6 \%$ of the patients (16).

If brain shift in a posterior direction, it is understood as the main cause of discrepancy between the planned and definitive electrode trajectory, a higher number of posterior trajectories and fewer anterior trajectories can be expected on the left side when compared to the side of the first implanted electrode. This assumption was not confirmed by the statistical evaluation. Therefore, the comparison of the final electrode position on the right and left sides does not support the role of brain shift as the most important causative factor for the difference between the anatomical and electrophysiological targets.

The observation of different definitive positions was supported by the results of Patel. The authors observed marked variations in subthalamic nucleus size and orientation. A significant difference was demonstrated when comparing left and right sided $\mathrm{x}$ and $\mathrm{y}$ coordinates. The same difference between right and left surgical trajectories observed in our study supported this data (25).
Structural changes of the subthalamic nucleus caused by the natural course of progressive neurodegenerative disease can be considered as another cause of the discrepancy between the anatomical target and final electrode placement. If this were true, a higher percentage of central-anatomical electrodes could be expected in younger patients and patients with shorter disease duration. However, no significant difference in age and disease duration was proven when the group of patients in whom central electrodes were implanted was compared to patients with other than central electrode implanted. Although the duration of L-DOPA adverse effects may not reflect the degree of degenerative disease progression, it should be noted that the statistical evaluation did not prove a significant difference of L-DOPA adverse effect duration between patients with central and non-central electrode implanted.

Finally, the length of subthalamic nucleus recording was compared between the group of patients with central electrodes and patients with non-central electrode implanted. The length of subthalamic activity in our study was slightly lower than that presented by other authors (Hamid et al, $4.65 \mathrm{~mm}$; McClelland III et al, $4.5 \mathrm{~mm}$ ) because of different methodologies for the subthalamic nucleus length determination and the use of medians in our study. The length of subthalamic activity may reflect more than the activity of the motor subthalamus (association and limbic subthalamic parts are located in the lower subthalamic nucleus) and the length of the subthalamic nucleus may also correlate with the surgical trajectory angle $(15,26)$.

The statistical analysis did not confirm any difference in subthalamic nucleus activity length between the central-anatomical final trajectory and other trajectories on the left side (implanted as second side), not supporting a possible role of brain shift in the difference between the anatomical and final electrode targets. Surprisingly for right electrodes, the statistical evaluation proved a significantly longer length of recorded subthalamic activity in non-central electrode locations.

Another important role of the electrophysiological monitoring is the intraoperative detection of surgical problems. The electrophysiological activity substantially differing from expected findings should draw an attention to possible surgical problems that may occur before surgery (e.g. incorrect target structure choice), during preparatory phase (e.g. stereotactic frame setting error, stereotactic frame dislocation) and intraoperatively (e.g. marked brain shift, intracerebral bleeding with electrophysiological silence) (27).

Data supporting the use of intraoperative monitoring, increasing a surgical invasiveness, should be confronted with possible surgical risks and benefits. The low incidence of haemorrhagic complications in our series $(1.7 \%)$ matches the data presented by other authors $(5,7)$, although the literature overview report by Hariz stated that microelectrode recordings were five times more likely associated with haemorrhagic complication (4).

\section{Conclusions}

Although the study criteria analysing the relationship of the anatomical targets and targets defined with the use of the electrophysiological monitoring may look simple (MicroDrive trajectory, 
subthalamic nucleus activity length), it provides new insights into the determination of possible factors responsible for the differences between the anatomical and functional targets. The differences cannot be attributed only to brain shift. The possible roles of age, Parkinson's disease duration, and L-DOPA adverse effects were not confirmed by the study results. Study results, together with a low incidence of haemorrhagic complications, provide the evidence supporting the use of the intraoperative microrecording for subthalamic electrode targeting.

\section{References}

1. Acar F, Miller JP, Berk MC, Anderson G, Burchiel KJ. Safety of anterior commissure-posterior commissure-based target calculation of the subthalamic nucleus in functional stereotactic procedures. Stereotact Funct Neurosurg 2007; 85 (6): 287-291.

2. Andrade-Souza YM, Schwalb JM, Hamani C et al. Comparison of three methods of targeting the subthalamic nucleus for chronic stimulation in Parkinson's disease. Neurosurgery 2008; 62 (Suppl 2): 875-873.

3. Ondo WG, Bronte Stewart H. The North American survey of placement and adjustement strategie for deep brain stimulation. Stereotact Funct Neurosurg 2005; 83 (4): 142-147.

4. Hariz MI. Safety and risk of microelectrode recording in surgery for movement disorders. Stereotact Funct Neurosurg 2002; 78 (3-4): 146-157.

5. Gorgulho A, De Salles AA, Frighetto L, Behnke E. Incidence of hemorrhage assoaciated with electrophysiological studies performed using macroelectrodes and microelectrodes in functional neurosurgery. $\mathrm{J}$ Neurosurg 2005; 105 (5): 888 -896.

6. Palur RS, Berk C, Schulzer M, Honey CR. A metaanalysis comparing comparing the results of pallidotomy performed using microelectrode recording or macroelectrode stimulation. J Neurosurg 2002; 96 (6): $1058-1062$.

7. Sansur CA, Frysinger RC, Pouratian $\mathbf{N}$ et al. Incidence of symptomatic haematoma after stereotactic electrode placement. J Neurosug 2007; 107 (5): 998-1003

8. Binder DK, Rau G, Starr PA. Haemorrhagic complications of microelectrode- guided deep brain stimulation. Stereotact Funct Neurosurg 2003; 80 (1-4): 28-31.

9. Maldonado IL, Roujeau T, Cif $\mathbf{L}$ et al. Magnetic resonance-based deep brain stimulation technique: a series of 478 consecutive implanted electrodes with no perioperative intracerebral hemorrhage. Neurosurgery 2009; 65 (Suppl 6): 196-201.

10. Starr PA, Martin JA, Ostrem JL, Talke P, Levesque N, Larson PS. Subthalamic nucleus deep brain stimulator placement using high-field interventional magnetic resonance imaging and a skull mounted aiming device : technique and application accuracy. J Neurosurg 2010; 112 (3): 479 -490.

11. Cuny E, Guehl D, Burbaud P, Gross C, Dousset V, Rougier A. Lack of agreement between direct magnetic resonance imaging and statistical determination of a subthalamic target: the role of electrophysiological guidance. J Neurosurg 2002; 97 (3): 591 - 597.

12. Forster A, Eljamel MS, Varma TR, Tulley M, Latimer M. Audit of neurophysiological recording during movement disorder surgery. Stereotact Funct Neurosurg 1999; 72 (2-4): 154-156.
13. Pinsker MO, Volkmann J, Falk D, Herzog J, Steigerwald F, Deuschl G ,Mehdorn HM. Deep brain stimulation of internal globus pallidus in dystonia : target localisation under general anaesthesia. Acta Neurochir (Wien) 2009; 151 (7): 751-758.

14. Amirnovin R, Williams ZM, Cosgrove GR, Eskandar EN. Experience with microelectrode guided subthalamic nucleus deep brain stimulation. Neurosurgery 2006; 58 (Suppl 1): 96-102.

15. Hamid NA, Mitchell RD, Mocroft P, Westby GW, Milner J, Pall H. Targeting the subthalamic nucleus for deep brain stimulation: technical approach and fusion of pre- and postoperative MR images to define accuracy of lead placement. J Neurol Neurosurg Psychiatry 2005; 76 (3): 409-414.

16. Bour LJ, Contarino MF, Foncke EM et al. Long-term experience with intraoperative microrecording during DBS neurosurgery in Stn and GPi. Acta Neurochir (Wien) 2010; 152 (12): 2069-2077.

17. Bejjani BP, Dormont D, Pidoux B et al. Bilateral subthalamic stimulation for Parkinson's disease by using three dimensional sterereotactic magnetic resonance imaging and electrophysiological guidance. J Neurosurg 2000; 92 (4): 615-625.

18. Taravari A, Traubner P, Gortha S, Petrov I, Petrova V, IsmajliMarku M. Clinical, neurophysiologic, neuropsychologic findings and short genetic analysis in patients with idiopathic Parkinson's disease. Bratisl Lek Listy 2010; 111 (3): 156-159.

19. Daniluk S, G Davies K, Ellias SA, Novak P, Nazzaro JM. Assessment of the variability in the anatomical position of the subthalamic nucleus among patients with advanced Parkinson's disease using magnetic resonance imaging. Acta Neurochir (Wien) 2010; 152 (2): 201-210.

20. Nunta -aree $S$, Tuntongtip D, Sitthinamsuwan B, Srirabheebhat $P$, Nitising A, Witthiwej T. Accuracy of various subthalamic nucleus targeting methods and an appropriated formula for Thai patients. J Med Assoc Thai 2010; 93 (9): 1043-1049.

21. Petersen EA, Holl EM, Martinez-Torres I et al. Minimizing brain shift in stereotactic neurosurgery. Neurosurgery 2010; 67 (Suppl Operative 3): 213-221.

22. Miyagi Y, Shima F, Sasaki T. Brain shift: an error factor during implantation of deep brain stimulation electrodes. J Neurosurg 2007; 107 (5): 989-997.

23. Elias WJ, Fu KM, Frysinger RC. Cortical and subcortical brain shift during stereotactic procedures. J Neurosurg 2007; 107 (5): 983-988.

24. Winkler D, Tittgemeyer M, Schwarz J, Preul C, Strecker K, Meixensberger $\mathbf{J}$. The first evaluation of brain shift during functional neurosurgery by deformation field analysis. J Neurol Neurosurg Psychiatry 2005; 76 (8): 1161-1163.

25. Patel NK, Khan SS, Gill SS. Comparison of atlas- and magneticresonance-imaging-based stereotactic targeting of the subthalamic nucleus in the surgical treatment of Parkinson disease. Stereotact Funct Neurosurg 2008; 86 (3): 153-161.

26. McClelland S 3rd, Ford B, Senatus PB et al. Subthalamic stimulation for Parkinson disease: determination of electrode location necessary for clinical efficacy. Neurosurg Focus 2005; 19 (5): E12.

27. Urgošík D, Jech R, Růžička E. Deep brain stimulation in patients with movement disorders- stereotactic surgery and intraoperative findings. Cesk Slov Neurol N 2011; 74/107 (2): 175-186. 